\title{
Phase III study of 5FU, etoposide and leucovorin (FELV) compared to epirubicin, cisplatin and 5FU (ECF) in previously untreated patients with advanced biliary cancer
}

\author{
S Rao ${ }^{1,2}$, D Cunningham ${ }^{*, 1,2}$, RE Hawkins ${ }^{3}$, ME Hill ${ }^{4}$, D Smith ${ }^{5}$, F Daniel ${ }^{6}$, PJ Ross ${ }^{1,2}$, J Oates ${ }^{1,2}$ and \\ AR Norman ${ }^{1,2}$
}

'Department of Medicine, Royal Marsden Hospital, Downs Road, Sutton, Surrey SM2 5PT, UK; ${ }^{2}$ Department of Medicine, Royal Marsden Hospital, London, UK; ${ }^{3}$ Christie Hospital, Manchester, UK; ${ }^{4}$ Kent Oncology Centre, Maidstone, UK; ${ }^{5}$ Clatterbridge Centre for Oncology, Clatterbridge, UK; ${ }^{6}$ Plymouth Oncology Centre, Plymouth, UK

The purpose of this study was to determine whether epirubicin, cisplatin and infused 5FU (ECF) improves overall survival (OS) compared to 5FU, etoposide and leucovorin (FELV) in patients with previously untreated advanced biliary cancer in a prospective randomised study. Patients were randomly assigned to receive epirubicin, cisplatin and infused 5FU ECF or bolus 5FU etoposide and leucovorin (FELV). The primary end point was OS with secondary end points of objective response rate (ORR), failure-free survival (FFS), quality of life (QOL) and toxicity. In all, 54 patients were recruited with 27 randomly assigned to each arm. The median OS for ECF was 9.02 months (95\% confidence interval (Cl): 6.46- II.5I) and FELV I 2.03 months (95\% Cl: 9.3- |4.7), P=0.2059. Objective response rates were similar for both arms: ECF 19.2\% (95\% Cl: 6.55-39.3); FELV I5\% (95\% Cl: 3.2-37.9), $P=0.72$. There was significantly increased grade $3 / 4$ neutropenia with FELV vs ECF (53.8 vs $29.5 \%$, respectively, $P=0.020$ ). Symptom resolution was impressive for both regimens. This is the largest reported randomised study to date in this setting. ECF did not improve OS compared to FELV, but was associated with less acute toxicity. These data suggest that chemotherapy can prolong OS and achieve good symptomatic relief in advanced biliary cancer.

British Journal of Cancer (2005) 92, 1650 - 1654. doi:I0.I038/sj.bjc.6602576 www.bjcancer.com

Published online 26 April 2005

(C) 2005 Cancer Research UK

Keywords: chemotherapy; advanced biliary cancer; ECF; FELV; untreated phase III

Biliary carcinoma is an uncommon malignancy, although its incidence appears to be increasing worldwide (Patel, 2001; TaylorRobinson et al, 2001; Davila and El Serag, 2002; Khan et al, 2002). The incidence in the United States is approximately 8 per million and the majority of patients are over 65 years of age. The mortality rates of biliary cancer correspond to the incidence, as the prognosis is very poor. Surgery remains the only potentially curative intervention; however, the majority of patients present late with advanced unresectable disease.

Overall there are relatively few published data evaluating palliative chemotherapy for unresectable disease. Glimelius et al randomised patients with advanced biliary and pancreatic cancer to best supportive care plus or minus 5FU, etoposide and leucovorin (FELV). The median survival for the chemotherapy arm was significantly higher compared to best supportive care alone for all patients $(6.5$ vs 2.5 months, $P<0.01)$, with a trend towards superior survival for the patients with biliary cancer (6.5 vs 2.5 months, $P=0.10$ ) (Glimelius et al, 1996). In addition, there was an improvement in overall quality of life (QOL) for those patients receiving chemotherapy. However, the chemotherapy group experienced considerable toxicity $-41 \%$ grades 3 and 4 .

*Correspondence: Professor D Cunningham;

E-mail: david.cunningham@icr.ac.uk

Revised 3 March 2005; accepted 17 March 2005; published online 26 April 2005
This was the only randomised trial reported in advanced biliary cancer at the time of designing our study. Several phase II studies had demonstrated activity with chemotherapy (both monotherapy and combination regimens), with response rates ranging from 0 to 34\% (Harvey et al, 1984; Taal et al, 1993; Jones et al, 1996; Patt et al, 1996; Ducreux et al, 1998). We conducted a phase II study in this setting evaluating the combination of epirubicin, cisplatin and infused 5FU (ECF) based on encouraging activity and tolerance in oesophagogastric cancer. This demonstrated an objective response rate (ORR) of $40 \%$, a median overall survival (OS) of 11 months and was associated with minimal grade $3 / 4$ toxicity (Ellis et al, 1995).

The lack of randomised studies in this setting, the encouraging results produced by FELV chemotherapy $v s$ best supportive care alone and the activity and tolerability of ECF in our phase II study led to the design of the randomised trial described in this report. The primary objective of this trial was to test whether ECF would improve the OS of patients with advanced biliary cancer compared to FELV chemotherapy.

\section{PATIENTS AND METHODS}

\section{Study conduct}

This multicentre randomised study was conducted in five centres in the United Kingdom. Signed informed consent was obtained 
from all patients prior to randomisation. The study was approved by the local institutional review boards at all participating centres.

\section{Main end points}

Overall survival was the primary end point. The secondary end points were ORR, failure-free survival (FFS), toxicity and the impact of treatment on QOL.

\section{Patient selection}

The main eligibility criteria were histologically or cytologically confirmed adenocarcinoma, squamous carcinoma or undifferentiated carcinoma of the gall bladder, intra/extrahepatic bile ducts or ampulla of Vater; no prior chemotherapy or radiotherapy; ECOG performance status (PS) $0-2$; neutrophils $>1.5 \times 10^{9} 1^{-1}$; platelets $>100 \times 10^{9} 1^{-1}$; total white cell count $>3.0 \times 10^{9} 1^{-1}$; total bilirubin $<30 \mathrm{mmoll}^{-1}$; glomerular filtration rate of $>60 \mathrm{ml} \mathrm{min}^{-1}$; and life expectancy $>3$ months.

Patients were excluded if there were medical or psychiatric conditions precluding informed consent or significant cardiac disease, arrythmias or angina pectoris.

\section{Randomisation and study treatment}

Eligible patients were centrally randomised electronically with stratification by centre. FELV comprised of $5 \mathrm{FU} 600 \mathrm{mg} \mathrm{m}^{-2}$ as intravenous (i.v.) bolus days $1-3$; etoposide $120 \mathrm{mg} \mathrm{m}^{-2}$ i.v. infusion over $40 \mathrm{~min}$ days $1-3$; and leucovorin $60 \mathrm{mg} \mathrm{m}^{-2}$ i.v. bolus days $1-3$. Each cycle was repeated every 3 weeks.

ECF comprised of $5 \mathrm{FU} 200 \mathrm{mg} \mathrm{m}^{-2}$ by continuous infusion via a central line for 24 weeks; epirubicin $50 \mathrm{mg} \mathrm{m}^{-2}$ day 1 ; and cisplatin $60 \mathrm{mg} \mathrm{m}^{-2}$ with hydration day 1 . Each cycle was repeated every 3 weeks.

\section{Dose modifications}

Toxicity was evaluated and graded according to the National Cancer Institute common toxicity criteria (version 2.0). Any grade $3 / 4$ nonhaematological toxicity resulted in treatment being withheld until recovery and then the following dose reductions were made: FELV 50\% dose reduction in 5FU subsequently; ECF $50 \%$ dose reduction of infusional 5FU for grade 3 and $75 \%$ for grade 4 . Any grade 3/4 haematological toxicity resulted in treatment being withheld until recovery and the following adjustments were made: FELV resumed at full dose for one delay, $25 \%$ dose reduction of 5FU and etoposide for 2 delays and $50 \%$ for more than 2 delays; ECF $25 \%$ dose reduction of epirubicin for grade 3 and $50 \%$ for grade 4 neutropenia, $50 \%$ for grade 3 and $75 \%$ for grade 4 thrombocytopenia.

\section{Safety evaluations}

Patients were assessed at baseline with a full medical history and physical examination including PS, full blood count, serum biochemistry including electrolytes, hepatic and renal function. A baseline EDTA or $24 \mathrm{~h}$ urinary clearance was performed. During the study, full medical history and physical examination including PS, blood count, serum biochemistry including electrolytes, hepatic and renal function were performed prior to each cycle of treatment.

\section{Efficacy evaluations}

Tumour response by CT assessment was performed according to WHO criteria at 12 and 24 weeks.

Overall survival and FFS were calculated for all randomised patients from the date of randomisation to the date of death or time to progression, respectively. Patients still alive were censored at the date of last contact.

\section{Quality of life}

Quality of life was assessed with the European Organisation for Research and Treatment of Cancer (EORTC) QLQ-C30 questionnaire (incorporating five function scales, one global health-status scale and nine symptoms scales), which was filled in at baseline, 6 , 12, 24 and 36 weeks.

\section{Statistics}

The primary end point was OS and the study was designed to detect a 1-year survival of $43 \%$ for ECF compared to $20 \%$ for FELV. Thus, a total of 116 patients ( 58 per arm) were required based on a two-sided $\alpha$ of $5 \%$ (overall power $=80 \%$ ). Overall survival and FFS were compared between treatment groups using a two-sided log-rank test, and survival was calculated from the date of randomisation to the date of death or the date of last follow-up. Failure-free survival was calculated from the date of randomisation until the date of progression/death or the date of last follow-up. For each treatment, Kaplan-Meier estimates of median survival and its $95 \%$ confidence interval (CI) were computed. To adjust for confounding variables, multivariate Cox's regression models were used. Tumour response rates and toxicity in the two arms were compared using the $\chi^{2}$ test, and Fisher's exact test was used where appropriate. The change from baseline in QOL was compared between the two treatment arms using the Mann - Whitney test. $P$ values of less than 0.05 were considered to be statistically significant and all analyses were performed by intention to treat.

\section{RESULTS}

Recruitment was slow and thus 27 patients were randomised to ECF and 27 to FELV between January 1997 and November 2003 in five centres in the United Kingdom. The patient baseline characteristics are shown in Table 1 and were generally well balanced between both groups, although there were more patients with bile duct carcinoma in the FELV group. The majority of patients were of PS $0-1$.

\section{Treatment}

The median duration of treatment was 5.5. cycles for ECF and six cycles for FELV. Dose reductions occurred in $18.5 \%$ of patients on ECF compared with $74 \%$ of patients receiving FELV. The main

Table I Demographics

\begin{tabular}{|c|c|c|}
\hline & ECF $(N=27)$ & FELV $(N=27)$ \\
\hline Median age (years) & 57 & 57 \\
\hline Age range (years) & $36-70$ & $39-76$ \\
\hline Male (\%) & 40.7 & 59.3 \\
\hline PS 0-I (\%) & 74.1 & 85.2 \\
\hline Metastatic & $16(59.3 \%)$ & 18 (66.7\%) \\
\hline \multicolumn{3}{|l|}{ Subsite } \\
\hline Ampulla & $6(22.2 \%)$ & $4(\mid 4.8 \%)$ \\
\hline Gall bladder & $14(51.9 \%)$ & $12(44.4 \%)$ \\
\hline Bile duct & 7 (25.9\%) & $11(40.7 \%)$ \\
\hline \multicolumn{3}{|l|}{ No. of disease sites } \\
\hline $0-1$ & 13 & 15 \\
\hline$\geqslant 2$ & 14 & 12 \\
\hline
\end{tabular}


Table 2 Objective response

\begin{tabular}{lcc}
\hline & ECF, $\boldsymbol{N}=\mathbf{2 6}$ (\%) & FELV, $\mathbf{N}=\mathbf{2 0}$ (\%) \\
\hline CR & $1(3.8)$ & - \\
PR & $4(15.4)$ & $3(15)$ \\
SD & $12(46.2)$ & $9(45)$ \\
PD & $9(34.6)$ & $8(40)$ \\
\hline
\end{tabular}

$E C F=$ epirubicin, cisplatin and 5FU; FELV $=5 F U$, etoposide and leucovorin; $\mathrm{CR}=$ complete response; $\mathrm{PR}=$ partial response; $\mathrm{SD}=$ stable disease; $\mathrm{PD}=$ progressive disease.

reason for dose reductions in the FELV arm was neutropenia. At least one dose delay occurred in $44.4 \%$ of patients in the ECF arm and $59.3 \%$ of patients in the FELV arm.

The dose intensity of each drug for both regimens is as follows: ECF epirubicin 88\%; cisplatin 90\%; 5FU 84\%: FELV etoposide $89 \%$; folinic acid $91.6 \%$; and $5 \mathrm{FU} 76 \%$. There was a trend towards a superior dose intensity of $5 \mathrm{FU}$ in the ECF cohort compared to FELV (84 vs $76 \%, P=0.056$ ).

\section{Tumour response and symptomatic resolution}

Eight patients were not evaluable for response: six had no radiologically measurable disease and two patients stopped treatment after only one cycle due to biliary stent infection and Hickman line infection, respectively.

Objective. response rates were similar for both arms (ECF 19.2\% (95\% CI: 6.6-39.3); FELV 15\% (95\% CI: 3.2-37.9), $P=0.999$ (Table 2)). A further proportion of patients in both arms achieved stable disease (ECF $46.2 \%$ compared to FELV $45 \%$ ). The rate of progressive disease was comparable between both groups (ECF $34.6 \%$ vs FELV $40 \%$ ).

Symptom resolution was achieved with both regimens ranging from 20 to $92 \%$ for a variety of symptoms including lethargy, pain, weight loss and anorexia (Table 3 ).

\section{Survival}

With a median follow-up of 387 days, there was no statistically significant difference in median OS: ECF 9.02 mths. (95\% CI: $6.46-$ $11.51)$ and FELV12.03 $\mathrm{mths}$ (95\% CI: 9.3-14.7), $P=0.2059$ (Figure 1). Multivariate analysis for OS confirmed these findings after controlling for performance status, locally advanced disease and alkaline phosphatase split on the median, there was no difference between arms, $P=0.096$. The 1 year OS for FELV was $50.2 \%$ (95\% CI: $30-67.3)$ vs $21 \%$ (95\% CI: $7.8-38.6)$ for ECF.

\section{Failure-free survival}

The median FFS for ECF was 157 days (95\% CI: 102.09-211. 9) and for FELV 220 days (95\%CI: $138-301.4)$. The 1 year FFS for FELV was $27.1 \%$ (95\% CI: 12 -44.7) vs $11.6 \%$ (95\% CI: $2.9-26.8)$ for ECF (Figure 2).

\section{Quality of life}

The compliance of patients in the QOL analysis was generally poor for both groups. The global QOL score at baseline for ECF and FELV: 62.9 vs 55.1 and at 12 weeks: 69.0 vs 70.83 .

\section{Toxicity}

The incidence of grade $3 / 4$ adverse events is shown in Table 4 . The non-haematological toxicity was similar between both groups aside from infection, which was significantly higher in the FELV arm. There was one treatment related death in the FELV arm due to sepsis and febrile neutropenia. There was a statistically higher

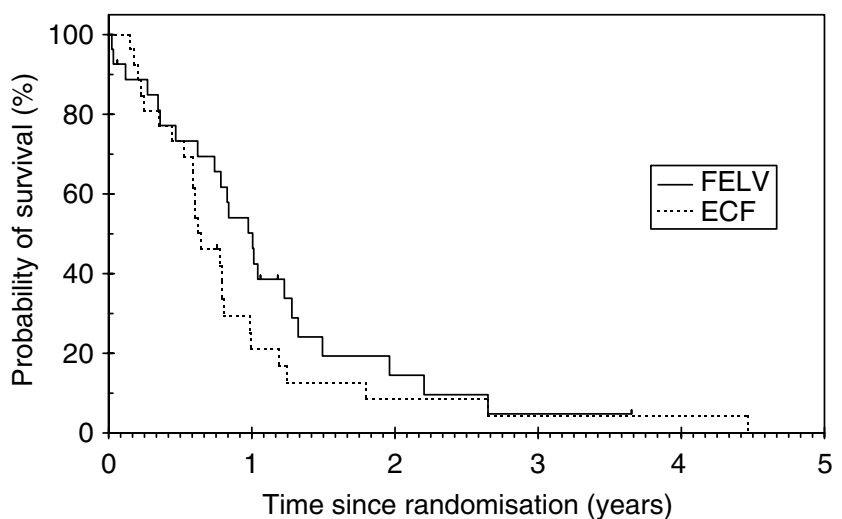

Figure I Overall survival (OS). With a median OS for ECF of 275 days (95\% Cl: $198-35 \mathrm{I}$ ) and 367 days for FELV (95\% Cl: $285-448)$, there was no statistically significant difference, $P=0.2059$.

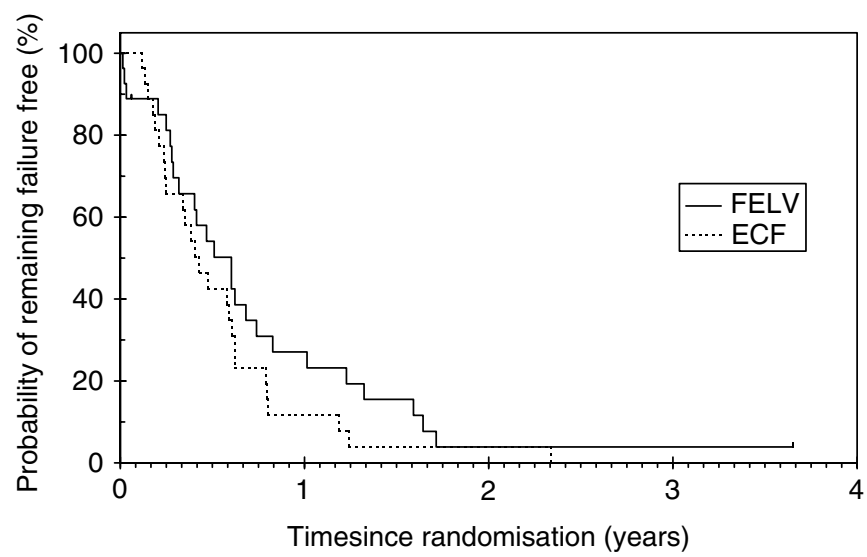

Figure 2 Failure-free survival (FFS). With a median FFS for ECF of 157 days (95\% Cl: |02.09-2| |.9) and for FELV 220 days (95\% Cl: | $38-30$ |.4), there was no statistically significant difference.

Table 3 Symptom resolution

\begin{tabular}{lcc}
\hline Symptom & ECF, N (\%) & FELV, $\mathbf{N}$ (\%) \\
\hline Pain & $12 / 1580$ & $14 / 1878$ \\
Anorexia & $11 / 1292$ & $7 / 1164$ \\
Weight loss & $9 / 1182$ & $7 / 978$ \\
Nausea & $2 / 367$ & $3 / 475$ \\
Lethargy & $6 / 1443$ & $3 / 1520$ \\
\hline
\end{tabular}

$\mathrm{ECF}=$ epirubicin, cisplatin and 5FU; FELV = 5FU, etoposide and leucovorin

incidence of grade 3/4 neutropenia for those patients receiving FELV compared to ECF (53.8 vs $29.5 \%$ respectively, $P=0.02$ ).

\section{Second line therapy}

Four patients (18.5\%). in the FELV arm went on to receive ECF on disease progression. 5 (18.5\%) patients in the ECF group underwent further chemotherapy (2: carboplatin and 5FU; 1: 5FU and mitomycin C; 1: ZD 9331 and gemcitabine; 1 patient was rechallenged with ECF.

\section{DISCUSSION}

At the time of designing this study there were relatively few published data for chemotherapy in advanced biliary cancer and 
Table 4 Toxicity

\begin{tabular}{|c|c|c|}
\hline & $E C F, N=25(\%)$ & FELV, $N=24(\%)$ \\
\hline Diarrhoea & $3(12)$ & $3(12.5)$ \\
\hline Stomatitis & $2(8)$ & $2(8.33)$ \\
\hline Nausea and vomiting & $4(16)$ & $2(8.33)$ \\
\hline Alopecia & $10(40)$ & $18(75)$ \\
\hline Infection & $4(16)$ & $10(41.7)$ \\
\hline \multirow{4}{*}{$\begin{array}{l}\text { Fever } \\
\text { Lethargy } \\
\text { Febrile neutropenia }\end{array}$} & $3(12)$ & $2(8.33)$ \\
\hline & $14(56)$ & $14(58.3)$ \\
\hline & & I (4.2) \\
\hline & $\mathbf{N}=\mathbf{2 7}(\%)$ & $\mathbf{N}=\mathbf{2 6}(\%)$ \\
\hline Anaemia & $7(25.9)$ & $3(\mid 1.5)$ \\
\hline Thrombocytopenia & I (3.7) & $2(8.3)$ \\
\hline Neutropenia & $7(25.9)$ & $14(53.8)$ \\
\hline
\end{tabular}

$E C F=$ epirubicin, cisplatin and 5FU; FELV =5FU, etoposide and leucovorin.

thus a pragmatic approach was employed to evaluate the activity of ECF in this setting. The statistical design of the study was based on previously published data for ECF and FELV. Unfortunately accrual was very slow leading to closure after only 54 patients were enrolled over six years. The poor recruitment in our study is a reflection of this difficult population who often present at an advanced stage and are thus unfit for chemotherapy. Thus due to slow accrual the study was not adequately powered to detect a meaningful difference in survival between the two arms. Nevertheless this study remains the largest randomised trial in this setting to date.

ECF produced similar ORR, symptom resolution and FFS to FELV and was associated with significantly less acute toxicity. There was no difference in OS with FELV compared to ECF (median OS: 12.03 mths $v s 9.02$ mths HR 1.43 (95\% CI: $0.81-2.84$ ), $P=0.2059)$.

This may be partly explained by $4(18.5 \%)$ patients in the FELV arm receiving second line chemotherapy with ECF on disease progression. However $5(18.5 \%)$ patients in the ECF group underwent further chemotherapy (2: carboplatin and 5FU; 1 : 5FU and mitomycin C; 1: ZD 9331 and gemcitabine; 1 patient was rechallenged with ECF) therefore this is unlikely to have accounted for such a difference.

A potential criticism of this study design is the absence of an observational control arm. There was a survival benefit for all patients (biliary and pancreatic cancer) receiving FELV chemotherapy $v s$ best supportive care in the Scandinavian randomised study (Glimelius et al, 1996). This difference was not significant when the biliary subset was analysed separately. However the study was only powered to detect a 6 months difference in OS with chemotherapy for all patients. Furthermore QOL was significantly improved for the chemotherapy subset, thus on balance we felt it reasonable to utilise FELV chemotherapy as the reference comparator arm in our trial.

Overall non-haematological toxicity was similar for both regimens. However there was significantly higher-grade 3/4 neutropenia and infection reported in the FELV arm. This resulted in sepsis and subsequent treatment related death in one patient. Myelosuppression was the main reason for treatment interruptions in the FELV group, this is reflected by the lower dose intensity of $5 \mathrm{FU}$ with this regimen compared to ECF (76 vs $84 \%, P=0.056$ ).

Quality of life data for all patients was generally sparse due to poor attrition. Thus it is difficult to draw any meaningful conclusions. There was no statistically significant difference between global QOL scores over time for both arms. Symptom resolution was impressive and comparable in both regimens particularly for pain, weight loss and nausea. This is encouraging for this group of patients who frequently present with multiple symptoms.

There have been 2 other randomised studies reported for advanced biliary cancer. The Scandinavian study of 37 patients demonstrated a median OS of 6 months for FELV chemotherapy compared to 2.5 months for best supportive care (Glimelius et al, 1996). An EORTC study of 53 patients compared high dose 5FU with or without cisplatin. The median OS for $5 \mathrm{FU}$ alone was 5.3 months compared to 7.8 months for the combination arm (Mitry et al, 2002). In our study of 54 patients the median OS for FELV was 12.03 months and 9.03 months for ECF. Thus our data are encouraging and provide supporting evidence that chemotherapy can prolong OS in this disease.

A variety of chemotherapeutic agents have been investigated in this setting mainly in the context of phase II trials. Gemcitabine is of particular interest based on its significant activity in pancreatic cancer (Burris et al, 1997). It has been used as monotherapy and in combination with capecitabine, irinotecan, cisplatin and $5 \mathrm{FU}$ among others. Objective response rates range from $8-39 \%$ and tolerance is generally acceptable. (Kubicka et al, 2001; Penz et al, 2001; Jani et al, 2002; Jacobson et al, 2003; Thongprasert et al, 2003; Knox et al, 2004). Thus further randomised studies are underway or planned with these combination regimens vs gemcitabine alone.

In conclusion ECF produced similar ORRs, FFS and symptom resolution to FELV with less acute toxicity. As a result of poor recruitment the study was underpowered to detect a significant difference in OS between the two regimens. Thus based on these data it is not possible to define a reference regimen for advanced biliary cancer. Nevertheless this study has shown that chemotherapy can prolong OS and provide good symptomatic relief for these patients. Further adequately powered randomised trials are required to establish the standard of care for this disease.

\section{REFERENCES}

Burris III HA, Moore MJ, Andersen J, Green MR, Rothenberg ML, Modiano MR, Cripps MC, Portenoy RK, Storniolo AM, Tarassoff P, Nelson R, Dorr FA, Stephens CD, Von Hoff DD (1997) Improvements in survival and clinical benefit with gemcitabine as first-line therapy for patients with advanced pancreas cancer: a randomized trial. J Clin Oncol 15: 2403-2413

Davila JA, El Serag HB (2002) Cholangiocarcinoma: the 'other' liver cancer on the rise. Am J Gastroenterol 97: 3199-3200

Ducreux M, Rougier P, Fandi A, Clavero-Fabri MC, Villing AL, Fassone F, Fandi L, Zarba J, Armand JP (1998) Effective treatment of advanced biliary tract carcinoma using 5-fluorouracil continuous infusion with cisplatin. Ann Oncol 9: 653-656

Ellis PA, Norman A, Hill A, O'Brien ME, Nicolson M, Hickish T, Cunningham D (1995) Epirubicin, cisplatin and infusional 5-fluorouracil (5-FU) (ECF) in hepatobiliary tumours. Eur J Cancer 31A: 1594-1598
Glimelius B, Hoffman K, Sjoden PO, Jacobsson G, Sellstrom H, Enander LK, Linne T, Svensson C (1996) Chemotherapy improves survival and quality of life in advanced pancreatic and biliary cancer. Ann Oncol 7: 593-600

Harvey JH, Smith FP, Schein PS (1984) 5-Fluorouracil, mitomycin, and doxorubicin (FAM) in carcinoma of the biliary tract. J Clin Oncol 2: $1245-1248$

Jacobson SD, Alberts SR, Mahoney MR (2003) Phase II trial of gemcitabine (Gem), 5-fluorouracil (5FU), and leucovorin (LV) in patients with unresectable or metastatic biliary (Bili) and gallbladder (GB) carcinoma: a North Central Cancer Treatment Group (NCCTG) study. Proceedings of the American Society of Clinical Oncology, Vol 22, p 275; Abstract 1102

Jani RC, Bhargava P, Stuart K (2002) Multicenter phase II trial of gemcitibine and irinotecan in patients with advanced or metastatic 
biliary cancer. Proceedings of the American Society of Clinical Oncology, Vol 21(2), p 125; Abstract 2313

Jones Jr DV, Lozano R, Hoque A, Markowitz A, Patt YZ (1996) Phase II study of paclitaxel therapy for unresectable biliary tree carcinomas. J Clin Oncol 14: 2306-2310

Khan SA, Davidson BR, Goldin R, Pereira SP, Rosenberg WM, TaylorRobinson SD, Thillainayagam AV, Thomas HC, Thursz MR, Wasan $\mathrm{H}$ (2002) Guidelines for the diagnosis and treatment of cholangiocarcinoma: consensus document. Gut 51(Suppl 6): VI1 - VI9

Knox JJ, Hedley D, Oza A (2004) Phase II trial of gemcitabine plus capecitabine (GemCap) in patients with advanced or metastatic biliary tract cancer. Proceedings of the American Society of Clinical Oncology, Vol 22, p 317; Absract 1275

Kubicka S, Rudolph KL, Tietze MK, Lorenz M, Manns M (2001) Phase II study of systemic gemcitabine chemotherapy for advanced unresectable hepatobiliary carcinomas. Hepatogastroenterology 48: 783-789

Mitry E, Van Cutsem E, Van Laethem JL (2002) A randomised phase II trial of weekly high dose 5FU (HD-FU) with and without folinic acid (FA) and cisplatin (P) in patients (pts) with advanced biliary tract carcinoma: the EORTC 40955 trial. Proceedings of the American Society of Clinical Oncology, Vol 21(1), p 134; Abstract 696
Patel T (2001) Increasing incidence and mortality of primary intrahepatic cholangiocarcinoma in the United States. Hepatology 33: 1353-1357

Patt YZ, Jones Jr DV, Hoque A, Lozano R, Markowitz A, Raijman I, Lynch P, Charnsangavej C (1996) Phase II trial of intravenous flourouracil and subcutaneous interferon alfa-2b for biliary tract cancer. J Clin Oncol 14: $2311-2315$

Penz M, Kornek GV, Raderer M, Ulrich-Pur H, Fiebiger W, Lenauer A, Depisch D, Krauss G, Schneeweiss B, Scheithauer W (2001) Phase II trial of two-weekly gemcitabine in patients with advanced biliary tract cancer. Ann Oncol 12: $183-186$

Taal BG, Audisio RA, Bleiberg H, Blijham GH, Neijt JP, Veenhof CH, Duez N, Sahmoud T (1993) Phase II trial of mitomycin C (MMC) in advanced gallbladder and biliary tree carcinoma. An EORTC Gastrointestinal Tract Cancer Cooperative Group Study. Ann Oncol 4: 607-609

Taylor-Robinson SD, Toledano MB, Arora S, Keegan TJ, Hargreaves S, Beck A, Khan SA, Elliott P, Thomas HC (2001) Increase in mortality rates from intrahepatic cholangiocarcinoma in England and Wales 1968-1998. Gut 48: $816-820$

Thongprasert S, Napapan S, Charoentum C (2003) Phase II study of gemcitabine plus cisplatin in biliary tract cancer. Proceedings of the American Society of Clinical Oncology, Vol 22, p 304; Abstract 1221 